\title{
Deconstructing pheromone-mediated behavior one layer at a time
}

\author{
Gabriela Sánchez-Andrade and Darren W Logan ${ }^{*}$
}

\begin{abstract}
The vomeronasal organ, a sensory structure within the nasal cavity of most tetrapods, detects pheromones that influence socio-sexual behavior. It has two neuronal layers, each patterned by distinct receptor sub-families coupled to different G-proteins. Work recently published in this journal found female mice with one layer genetically inactivated are deficient in a surprisingly wide range of reproductive behaviors, providing new insights into how the nose can influence the brain.

See research article:

http://www.biomedcentral.com/1741-7007/12/31
\end{abstract}

\section{Commentary}

All animals secrete or excrete complex chemical signatures into the external environment: in sweat, tears, urine, feces or in scent marks from specialized exocrine glands. These have the potential to provide information on the identity, sex, age, fitness and threat afforded by the animal that deposited them. Consequently, many species have evolved to use these for inter-individual communication. One of the sensory subsystems that detect such chemicals is the vomeronasal organ (VNO), a highly specialized neuroepithelium found in the base of the nose of most tetrapods. The VNO is responsible for detecting intra-specific and inter-specific chemical signals, known as pheromones and kairomones, respectively, that initiate innate behaviors [1].

The VNO epithelium is organized into two cellular layers (Figure 1). Neurons in the apical layer express a receptor from the V1R family and the Goi2 G-protein, which are together thought to initiate signal transduction after the receptor binds a semiochemical ligand. Those in the basal layer express V2R receptors and the Goo G-protein, which are likely to fulfill a similar function [2]. Neurons in both layers express a member of the transient receptor potential

\footnotetext{
* Correspondence: dl5@sanger.ac.uk

Wellcome Trust Sanger Institute, Hinxton, Cambridge CB10 1SA, UK
}

family of ion channels, TRPC2, a downstream component of both signal transduction pathways [3]. V1R/Gai2 and $\mathrm{V} 2 \mathrm{R} / \mathrm{G} \alpha \mathrm{o}$ neurons project to different aspects of the accessory olfactory bulb in the brain; thus, the two layers of the VNO may transmit very different types of information. While significant progress has been made towards defining the source and chemical characteristics of the ligands that bind V1R and V2R receptors in mice [2], the sufficiency and necessity of each neuronal layer in instructing differential behavior are still largely unknown. This is due, in part, to the complex genetic engineering involved in generating animals that have one layer entirely and exclusively inactivated [4]. In this journal, Oboti et al. report an extremely comprehensive analysis of sexual and reproductive behavior in mice with a non-functional V2R/Goo layer [5]. This new work, along with a companion study of aggressive behavior in the same mutant line [6], marks the first step towards deconstructing the behavioral logic encoded within the layers of the VNO.

\section{Behavioral consequences of layer specific inactivation}

Goi2 and Goo have been independently ablated in mice previously $[7,8]$. Although both mutant lines do display abnormal behavior, the G-proteins are expressed in other tissues so the precise role of the VNO in the behavioral phenotype could not be determined. To resolve this, Oboti and colleagues applied the Cre-Lox recombinase system to delete Goo only in cells that express olfactory marker protein (OMP). Although these genes are co-expressed in some other sensory neurons in the nose, albeit at lower levels, only those in the basal VNO layer show a significant reduction in Goo expression [6].

When tested in a battery of assays, a remarkably large range of behavioral and physiological deficiencies are observed in these mice (Figure 1). For example, male urine is unable to induce estrus in adult mutant females (a phenomenon known as the Whitten effect) while juveniles fail to undergo puberty acceleration in response to male urinary cues (the Vandenbergh effect). Adult female 


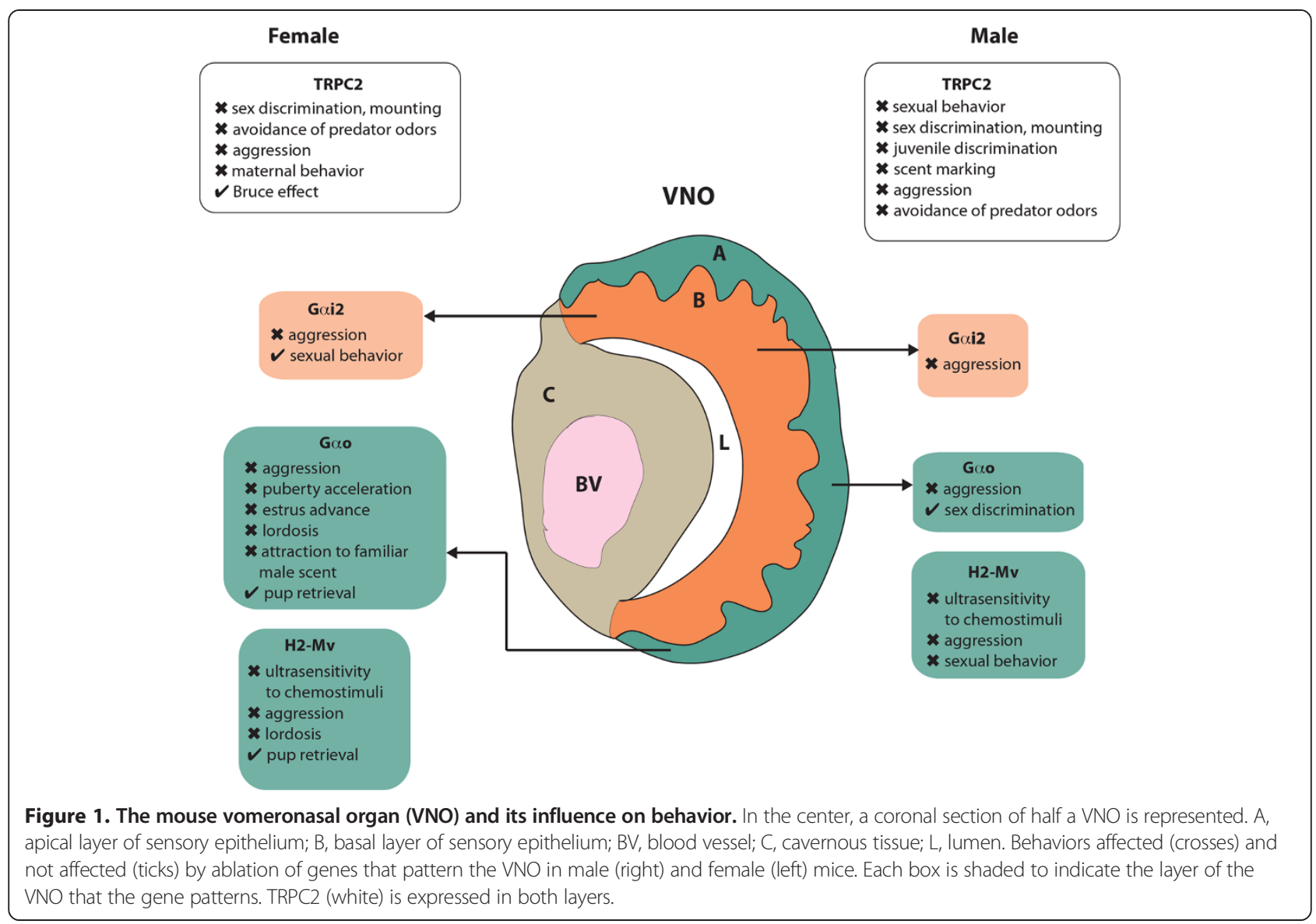

mice also fail to adopt a characteristic mating stance when exposed to male suitors, and are unable to detect and remember male-specific non-volatile individuality cues [5]. In addition, both mutant males and postpartum mothers display severely curtailed aggressive responses to intruders [6]. These phenotypes are consistent with the loss of acute pheromone signaling through V2Rs (coupled to Goo) in the basal layer of the VNO. However, similar behavioral abnormalities have been reported in Trpc2-/- mice, which have both VNO layers inactivated [2]. Does this imply the apical layer of the VNO has no role in mediating aggressive or sexual behaviors? We consider this unlikely, as there are aggression-promoting semiochemicals in male urine that activate the V1R/Gai2 layer exclusively [9]. It is possible that deactivating one layer could have an indirect effect on the function of the other. However, mice lacking basal layer function do display some behavioral differences to those with the whole VNO genetically inactivated, suggesting the Goi2 layer is at least partly functional. A more feasible explanation may be that parallel signaling through both layers is required to instruct certain behaviors. Although initially segregated, evidence suggests that circuits downstream of $V 1 R$ and $V 2 R$ neurons converge deeper in the brain where synergistic activation could be necessary to generate a behavioral response.

\section{Is the VNO more than a pheromone sensor?}

Interrupting signal transduction in the VNO appears to have consequences beyond acute pheromone detection. $\operatorname{Trpc} 2-1-$ mice have a reduced number of neurons in the VNO, suggesting its activity is important for cellular maintenance [3]. The basal VNO layer is also partially degenerated in conditional Goo mutant mice, while the apical layer remains unaffected [6]. What phenotypic impact might a reduction in the number of neurons in the basal layer have on the mice? Curiously, group-housed conditional Goo mutant females have severely disrupted estrus cycles, suggesting the basal layer of the VNO influences reproductive physiology independent of male pheromone signaling [5]. The mechanism through which this occurs is unclear. Gonadotropin releasing hormone $(\mathrm{GnRH})$ neurons migrate from the VNO to the forebrain during early development, where they control the release of reproductive hormones. It is therefore tempting to speculate that a degenerated basal layer could interfere with $\mathrm{GnRH}$ neuron migration or function. However, circulating reproductive hormone levels and 
ovary morphology appear normal in adult mice and Trpc2-/- females, with degeneration in both VNO layers, are not reported to have irregular estrus cycles [5]. This unexpected phenotype may hint at a more complex relationship between the $\mathrm{VNO}$ and reproductive physiology than previously thought.

\section{Future directions in understanding VNO function}

Producing mice with other subsets of VNO neurons inactivated is becoming ever easier due to technological advances in genetic engineering [4]. Indeed, another recent study used a sophisticated chromosome engineering approach to further functionally subdivide the V2R/Goo layer [10]. Members of the $H 2-M v$ cluster, a family of nonclassical class I major histocompatibility complex genes, are expressed in approximately half of the cells in the basal layer of the VNO. When these genes are deleted the pheromone sensitivity of the neurons that express them is significantly decreased, but not entirely lost [10]. This results in both sexual and aggressive deficits even though two-thirds of the VNO remains unperturbed, further supporting a synergistic model of pheromone signaling.

Ultimately, the systematic gene targeting of over $500 \mathrm{~V} 1 \mathrm{R}$ and V2R genes will be necessary to reveal the full extent of the behavioral logic encoded within the molecular organization of the VNO, though, even in the era of largescale knockout mouse programs, this will be a significant undertaking [4]. In the meantime, increasingly sensitive transcriptomic analyses are likely to reveal genes that pattern the olfactory neurons of the nose in smaller and smaller sub-divisions. Conditionally deleting these in the VNO of mice should continue to provide novel insights into the pheromone-mediated behaviors of mammals.

\section{Acknowledgements}

The authors are supported by the Wellcome Trust, grant number 098051.

Published: 29 May 2014

\section{References}

1. Wyatt TD: Pheromones and Animal Behaviour: Chemical Signals and Signature Mixes. 2nd edition. Cambridge University Press; 2014.

2. Ibarra-Soria X, Levitin MO, Logan DW: The genomic basis of vomeronasalmediated behaviour. Mamm Genome 2014, 25:75-86.

3. Stowers L, Holy TE, Meister M, Dulac C, Koentges G: Loss of sex discrimination and male-male aggression in mice deficient for TRP2. Science 2002, 295:1493-1500.

4. van der Weyden L, White JK, Adams DJ, Logan DW: The mouse genetics toolkit: revealing function and mechanism. Genome Biol 2011, 12:224.

5. Oboti L, Pérez-Gómez A, Keller M, Jacobi E, Birnbaumer L, Leinders-Zufall T, Zufall F, Chamero P: A wide range of pheromone-stimulated sexual and reproductive behaviors in female mice depend on $\mathrm{G}$ protein $\mathrm{G}($ alpha)o. BMC Biol 2014, 12:31.

6. Chamero P, Katsoulidou V, Hendrix P, Bufe B, Roberts R, Matsunami H, Abramowitz J, Birnbaumer L, Zufall F, Leinders-Zufall T: G protein G(alpha)o is essential for vomeronasal function and aggressive behavior in mice. Proc Natl Acad Sci U S A 2011, 108:12898-12903.

7. Norlin EM, Gussing F, Berghard A: Vomeronasal phenotype and behavioral alterations in G alpha i2 mutant mice. Curr Biol 2003, 13:1214-1219.
8. Jiang M, Gold MS, Boulay G, Spicher K, Peyton M, Brabet P, Srinivasan Y, Rudolph U, Ellison G, Birnbaumer L: Multiple neurological abnormalities in mice deficient in the G protein Go. Proc Natl Acad Sci U S A 1998, 95:3269-3274.

9. Chamero P, Marton TF, Logan DW, Flanagan K, Cruz JR, Saghatelian A, Cravatt BF, Stowers L: Identification of protein pheromones that promote aggressive behaviour. Nature 2007, 450:899-902.

10. Leinders-Zufall T, Ishii T, Chamero P, Hendrix P, Oboti L, Schmid A, Kircher S, Pyrski M, Akiyoshi S, Khan M, Vaes E, Zufall F, Mombaerts P: A family of nonclassical class I MHC genes contributes to ultrasensitive chemodetection by mouse vomeronasal sensory neurons. J Neurosci 2014, 34:5121-5133.

doi:10.1186/1741-7007-12-33

Cite this article as: Sánchez-Andrade G and Logan DW: Deconstructing pheromone-mediated behavior one layer at a time. BMC Biology 2014 12:33. 